Vol. 38 (1988) [263-266]

\title{
A TOPOLOGICAL ZERO-ONE LAW FOR OPEN CONTINUOUS MAPS
}

\author{
E. BARONE AND K.P.S. BHASKara RAO
}

\begin{abstract}
We obtain a topological zero-one law for sets with the Baire property which are invariant under a semigroup of open continuous maps acting on a topological space.
\end{abstract}

\section{INTRODUCTION}

In [1] a topological zero-one law was obtained for sets with the Baire property which are invariant under a group of homeomorphisms. Generalising the results of $[1]$, in [2] another topological zero-one law was obtained for sets with the Baire property which are invariant under an equivalence relation. The purpose of the present paper is to generalise the results of $[\mathbf{1}]$ in a different direction, namely, we obtain a topological zero-one law for sets with the Baire property which are invariant under a semigroup of open continuous maps acting on a topological space.

For unexplained notions see $[1,2,5$ and 6$]$.

\section{THE MAIN RESULT}

We denote by $\mathbf{S}$ a semigroup (under composition) of open continuous maps from a topological space $X$ to itself.

THEOREM. Consider the statements:

(1) There is a point $x$ in $X$ for which $\{S(x) ; S \in \mathbf{S}\}$ and $\bigcup\left\{S^{-1}(x) ; S \in \mathbf{S}\right\}$ are dense in $X$.

(2) For any two nonempty open sets $U$ and $V$, there is an $S$ in $\mathbf{S}$ such that $S(U) \cap V \neq \emptyset$.

(2') For any two nonempty open sets $U$ and $V$, there is an $S$ in $\mathbf{S}$ such that $S^{-1}(U) \cap V \neq \emptyset$.

(3) Any nonempty open set $U$ for which $S(U) \subseteq U$ for every $S$ in $\mathrm{S}$, is dense in $X$.

(3') Any nonempty open set $U$ for which $S^{-1}(U) \subseteq U$ for every $S$ in $\mathbf{S}$, is dense in $X$.

(4) For any subset $D$ of $X$ with the Baire property for which $S^{-1}(D) \subseteq D$ for every $S$ in $\mathbf{S}$, either $D$ or $X-D$ is of first category in $X$.

Received 30 November 1987

Work supported by M.P.I. and C.N.R., done while the second Author was visiting University of Lecce

Copyright Clearance Centre, Inc. Serial-fee code: 0004-9729/88 \$A2.00+0.00. 
Then

(1) $\Rightarrow(2) \Leftrightarrow\left(2^{\prime}\right) \Leftrightarrow(3) \Leftrightarrow\left(3^{\prime}\right) \Rightarrow(4)$

If $X$ is a Baire space then (4) $\Rightarrow(2)$.

If further $X$ has a countable pseudo-base, then $(4) \Rightarrow(1)$.

PROOF :

$(1) \rightarrow(2)$ : Let $x$ be a point satisfying (1). If $U$ is a nonempty open set, we can find an $S_{1}$ in $\mathrm{S}$ and a point $y$ in $U$ for which $S_{1}(y)=x$. If $V$ is another nonempty open set, we can also find an $S_{2}$ in $\mathbf{S}$ for which $S_{2}(x) \in V$. Now $S_{2}\left(S_{1}(y)\right)$ is in $V$ and $y$ is in $U$. So we have $S_{2} S_{1}(U) \cap V \neq \emptyset$.

(2) $\Leftrightarrow\left(2^{\prime}\right)$ :

Let us observe that $S(U) \cap V \neq \emptyset \Leftrightarrow U \cap S^{-1}(V) \neq \emptyset$.

If $S(u) \cap V \neq \emptyset$ then let $y \in S(U) \cap V$. Let $x \in U$ be such that $S(x)=y$. Since $y \in V$, we have that $x \in S^{-1}(V)$. Hence $U \cap S^{-1}(V) \neq \emptyset$. Conversely, if $x \in U \cap S^{-1}(V)$ then $S(x) \in S(U)$ and $S(x) \in V$, so that $S(U) \cap V \neq \emptyset$.

$(2)=(3)$;

If $U$ is a set as in (3) and if $V$ is any nonempty open set, then from (2) we have $U \cap V \supset S(U) \cap V$ for every $S$ in $\mathbf{S}$ and for some $S$ in $\mathbf{S} S(U) \cap V \neq \emptyset$. Hence $U$ is dense in $X$.

$(3) \Rightarrow(2)$ :

If $U$ and $V$ are nonempty open sets and we define $U_{0}=\bigcup\{S(U) ; S \in \mathbf{S}\}$ then $U_{0}$ is an open set satisfying condition (3) and so $U_{0}$ is dense in $X$. Hence $U_{0} \cap V \neq \emptyset$ and this tells us that there is an $S$ in $\mathbf{S}$ such that $S(U) \cap V \neq \emptyset$.

$\left(2^{\prime}\right) \Leftrightarrow\left(3^{\prime}\right)$ :

can be proved similarly to $(2) \Leftrightarrow(3)$.

$(3) \Rightarrow(4)$ :

Suppose that $D$ is a subset of $X$ with the property of Baire for which $S^{-1}(D) \subset D$ for every $S$ in S. Let $D=U \triangle P$ where $U$ is open in $X$ and $P$ is of first category in $X$. If $D$ is not of first category, then $U$ is of second category and hence nonempty. Since $D=U \triangle P$, we have $(X-D) \cap U \subset P$. For every $S$ in $\mathbf{S}$ :

$$
S^{-1}(X-D) \cap S^{-1}(U) \subset S^{-1}(P)
$$

and the later set is of first category since $S$ is an open continuous map. Since $S^{-1}(D) \subset$ $D$, we have that $X-D \subset S^{-1}(X-D)$ and hence $(X-D) \cap S^{-1}(U) \subset S^{-1}(P)$. Thus $(X-D) \cap S^{-1}(U)$ is of first category for every $S$ in S. By $(3) U^{\prime}=\bigcup\left\{S^{-1}(U) ; S \in \mathbf{S}\right\}$ is an open dense set in $X$. Now $X-D \subset\left((X-D) \cap U^{\prime}\right) \cup\left(X-U^{\prime}\right)$. By the Banach category Theorem [6, Theorem 16.1, p. 62] $(X-D) \cap U^{\prime}$ is of first category and by (3) $X-U^{\prime}$ is nowhere dense. Thus $X-D$ is of first category.

$(4) \rightleftharpoons\left(2^{\prime}\right)$ : 
Let $X$ be a Baire space and let $U$ and $V$ be two nonempty open subsets of $X$. The subset $U^{\prime}:=\bigcup\left\{S^{-1}(U) ; S \in \mathbf{S}\right\}$ is a nonempty open set such that $S^{-1}\left(U^{\prime}\right) \subset U^{\prime}$ for every $S$ in S. By (4) $U^{\prime}$ or $X-U^{\prime}$ is of first category in $X$. By the fact that $X$ is a Baire space it follows that $X-U^{\prime}$ is of first category in $X$ and then that $U^{\prime}$ is dense in $X$. Hence $S^{-1}(U) \cap V \neq \emptyset$ for some $S$ in $\mathbf{S}$. $(4) \Rightarrow(1)$ :

Let $X$ be a Baire space with a countable pseudo-base. Then consider the sets $A=$ $\{x:\{S(x) ; S \in \mathbf{S}\}$ is dense in $X\}$ and $B=\left\{x: \bigcup\left\{S^{-1}(x) ; S \in \mathbf{S}\right\}\right.$ is dense in $\left.X\right\}$. We can show easily that the two sets are countable intersections of dense open sets. Then $A \cap B$ is also countable intersection of dense open sets. Hence $A \cap B \neq \emptyset$. This is exactly what we need. Look also at remark $E$ below.

\section{REMARKS AND EXAMPLES}

A. If $\mathbf{S}$ is a group the theorem reduces to the Theorem of [1].

B. Our theorem is of course comnected with [3] in the case $\mathbf{S}$ is a group and $X$ is a complete metric space.

C. A better version of this theorem can be obtained using the techniques of [2].

D. An appropriate version of this theorem to give only:

(4') For any subset $D$ of $X$ with the property of Baire which is invariant (that is $S^{-1}(D)=D$ for all $S$ in S), either $D$ or $X-D$ is of first category in $X$, in place of (4) can be formulated and proved.

E. Since for any set $D$ and for any $S$ in $\mathbf{S}$ :

$$
S(D) \subset D \Leftrightarrow D \subset S^{-1}(D) \Leftrightarrow S^{-1}(X-D) \subset X-D
$$

" $S^{-1}(D) \subset D$ " in (4) can be replaced by " $S(D) \subset D$ " or " $D \subset S^{-1}(D)$ ".

However if $D$ is a subset of $X$ with the Baire property, for which $D \subset S(D)$ for all $S$ in $\mathbf{S}$, neither $D$ nor $X-D$ need be of first category in $X$. For example, consider the shift transformation $S^{\prime}$ on the unilateral product of countably many copies of the real line equipped with its usual topology. If $\mathbf{S}$ is the semigroup generated by $S^{\prime}$ and if $D=(0,1) \times \mathbf{R} \times \mathbf{R} \times \ldots$ then $D \subseteq S(D)$ for all $S$ in $\mathrm{S}$, whereas neither $D$ nor its complement is of first category. However (2) is satisfied for this set-up.

F. The theorem still remains true (with the same proof), even if $S$ is a semigroup of continuous feebly open (that is $S(U)$ contains a nonempty open set for every nonempty open set $U$ ) maps.

G. Since condition (1) of the theorem is in two parts, we give two examples to show that neither part implies the other. 
Let $X=\{1,2,3, \ldots\}$ equipped with the discrete topology and $S: X \rightarrow X$ be the map defined by $S(x)=1$ for all $x$ in $X$. Then $S=\{S\}$ is a semigroup of open continuous maps for which $S^{-1}(1)$ is dense in $X$. Also there is no $x$ in $X$ for which $\{S(x) ; S \in S\}$ is dense in $X$. For this set up (2) is not true.

Let $X=\{1,2,3, \ldots\}$ equipped with the discrete topology and $S_{n}$ be defined by $S_{n}(x)=x$ if $x \neq 1$ and $S_{n}(1)=n$. Then $\mathbf{S}=\left\{S_{n}, n \in \mathrm{N}\right\}$ is a semigroup of open continuous maps and $\{S(1) ; S \in S\}$ is dense in $X$. Also there is no $x$ in $X$ for which $\bigcup\left\{S^{-1}(x) ; S \in S\right\}$ is dense in $X$. For this set up (2) is not true.

H. Probably an anlogue of this theorem can be proved in the setup of Morgan II [4].

\section{REFERENCES}

[1] M. Bhaskara Rao and K.P.S. Bhaskara Rao, 'A category analogue of the Hewitt-Savage zero-one law', Proc. Amer. Math. Soc. 44 (1973), 497-499.

[2] P.S. Bhaskara Rao and R. Pol, 'Topological zero-one laws', Coll. Math. 39 (1978), 13-23.

[3] K. Kuratowski, 'On the concept of strongly transitive systems in topology', Ann. Mat. Pura Appl. 48 (1974), 357-363.

[4] J.C. Morgan II, 'On zero-one laws', Proc. Amer. Math. Soc. 62 (1977), 353-358.

[5] J.C. Oxtoby, 'Cartesian products of Baire spaces', Fund. Math. 49 (1964), 157-166.

[6] J.C. Oxtoby, Measure and Category (Springer, Berlin, 1971).

Professor E. Barone

Dipartimento di Matematica

via Arnesano

73100 Lecce

Italy

\author{
Professor K.P.S. Bhaskara Rao \\ Indian Statistical Institute \\ Bangalore Centre \\ R.V. College Post \\ Bangalore 560059 \\ India \\ and \\ Department of Mathematics \\ University of Brunei Darussalam \\ Bandar Sri Bepanian \\ Brunei
}

